\title{
Can we predict relapse in Graves' disease? Results from a systematic review and meta-analysis
}

\author{
Tristan Struja 1, *, Hannah Fehlberg', *, Alexander Kutz', Larissa Guebelin', \\ Christian Degen', Beat Mueller, ${ }^{1,2}$ and Philipp Schuetz ${ }^{1,2}$ \\ ${ }^{1}$ Medical University Department, Clinic for Endocrinology, Diabetes \& Metabolism, Kantonsspital Aarau, \\ Aarau, Switzerland and ${ }^{2}$ Medical Faculty of the University of Basel, Basel, Switzerland \\ *(T Struja and H Fehlberg contributed equally to this work)
}

Correspondence should be addressed to T Struja

Email

tristan.struja@gmail.com

\begin{abstract}
Background: Identification of pretreatment risk factors predicting relapse in patients with hyperthyroidism of Graves' disease after stopping anti-thyroid drugs (ATD) is decisive to guide therapeutic options.

Purpose: We performed a systematic search and meta-analysis to study predictors for relapse after stopping ATD in patients with Graves' disease.

Methods: Based on a pre-specified protocol, we searched PubMed, EMBASE and Cochrane in July 2015 for casecontrol, controlled and randomized-controlled trials reporting risk factors for relapse after stopping ATD. The primary endpoint was relapse of disease until follow-up. PRISMA and SIGN statements were used for reviewing the data and assessing the quality of included trials.

Results: We included 54 trials with a total of 7595 participants. Most trials were small with moderate-to-high risk for bias. Ten trials were assessed only qualitatively (2227 patients), genomic data were reported in 13 trials (2178 patients) and 31 trials (4346 patients) were assessed quantitatively. In total, there were 3696 relapses in 7595 patients (48.7\%). By using random-effects meta-analysis, orbitopathy, smoking, thyroid volume measured by sonography, goiter size, fT4, TT3, TRAb and TBII were significantly associated with relapse, whereas male vs female sex, age and initial tT4 level
\end{abstract} did not show significant associations.

Conclusions: This analysis found several risk factors to predict relapse in Graves' disease, which can be combined in a risk score. Prospective studies should evaluate the prognostic accuracy of such a score to guide treatment decisions.

\section{Introduction}

Graves' disease is the most common cause of primary hyperthyroidism in iodine-sufficient areas. The overall prevalence of Graves' disease varies but is reported to be around $0.5 \%$ (1). Graves' disease is characterized by the autoimmune production of thyrotropin-related antibodies (TRAb) stimulating the thyroidal cells and causing an overproduction of thyroid hormones (2). Furthermore, up to $50 \%$ of patients develop Graves' orbitopathy by the co-expression of TSH receptors by pre-adipocytes within the orbital cavities inducing proliferation and thus proptosis (3).
() 2017 European Society of Endocrinology Printed in Great Britain
European Journal of Endocrinology

(2017) 176, 87-97 
with higher doses. Due to the high risk of relapse, an alternative first-line treatment used (mostly in the United States) is radioactive iodine (RAI) ablation, a usually ablative treatment modality $(3,6)$.

Several risk factors predicting relapse after stopping ATD have been reported, e.g. younger age, large goiters, smoking, male sex, severe biochemical disease and higher levels of antibodies (4). Still, there is a lack of a relapse risk prediction rule, which could be helpful when counseling patients about the best first treatment option (i.e. ATD, surgery or RAI), subjecting them to a potentially unnecessary and time-consuming ATD treatment. Herein, we performed a systematic review and meta-analysis to study the possible pretreatment risk factors of relapse of patients with hyperthyroidism due to Graves' disease.

\section{Methods}

\section{Objective}

The objective was to study pretreatment risk factor of disease recurrence after stopping ATD in patients with a first episode of hyperthyroidism due to Graves' disease.

\section{Protocol and eligibility criteria}

This review adheres to PRISMA guidelines (7). First, we generated a review protocol (Supplementary file, see section on Supplementary data given at the end of this article), outlining the main hypothesis, outcomes and search strategy. We included case-control, observational, randomized-controlled and controlled trials that assigned patients with a first episode of Graves' disease to a standard treatment with any ATD. The diagnosis of Graves' disease had to be established by a low thyrotropin (TSH), a high free or total thyroxine (f/tT4) or triiodothyronine (f/tT3), and if available, diffuse goiter and positive TRAb. Participants had to be adults of at least 16 years of age. There were no restrictions on the type and dosage of ATD used (e.g. methimazole, carbimazole, propylthiouracil, etc.). Also, we had no restrictions regarding language of publication, publication type or date and publication status. If studies were not available in a public library, we contacted the corresponding author. We also did include trials assessing the addition of T4 or T3 to standard ATD treatment as a former systematic review has shown that this does not affect the recurrence rate (6).

We excluded trials with a follow-up of $<12$ months, as well as case reports, reviews and trials not reporting relapse or risk factors, our primary endpoints of interest.

\section{Outcomes}

The primary outcome was relapse of hyperthyroidism according to pretreatment risk factors within a follow-up of at least 12 months after stopping ATD. We did not define any secondary outcomes.

\section{Search strategy and study selection}

We searched the electronic databases PubMed/Medline, EMBASE and the Cochrane CENTRAL Library (all until end of July 2015). We also hand-searched the references of leading articles to identify further articles.

Our search strategy included following terms: Graves' disease, relapse, recurrence, goiter, hyperthyroidism, Basedow (Supplementary file for further details). The articles found were transferred into EndNote Version X7 (Thomson Reuters, Philadelphia, PA, USA) and then screened by title and abstract. Full articles were retrieved for further assessment if the information given indicated that the study included patients with their first episode of Graves' disease, reported pretreatment risk factors and had well-defined criteria for the diagnosis of Graves' disease and relapse. If there was any doubt about the relevance of the article from the information given in the title and abstract, the full article was consulted for clarification. All articles were reviewed by two independent authors (HF and TS). In case of disagreements, a solution was obtained by consensus and if not possible by a third reviewer (PS) (Fig. 1).

\section{Data collection}

For all included studies, a data extraction table was generated as suggested in the Cochrane Handbook for Systematic Reviews of Interventions (8). It was then tested on two pilot studies and refined afterward. Data were extracted by two authors (HF and CD) and crosschecked by another researcher (TS). Two review authors independently assessed trial quality (HF and TS). In case of disagreements, a solution was again obtained by consensus and if not possible by a third reviewer (PS). We suspected duplicate publications if the same authors published multiple studies on the same subject within two years. In November 2015, we tried to contact 12 trial authors $(9,10,11,12,13,14,15,16,17,18,19,20)$ for suspected double publication of data and/or for missing data respectively. Four authors could not be contacted as there were no recent email addresses $(15,17,19,20)$. Two authors refused to share their data with us $(10,16)$. Until the end of February 2016, we received no answers from the other authors, although we contacted them twice. 


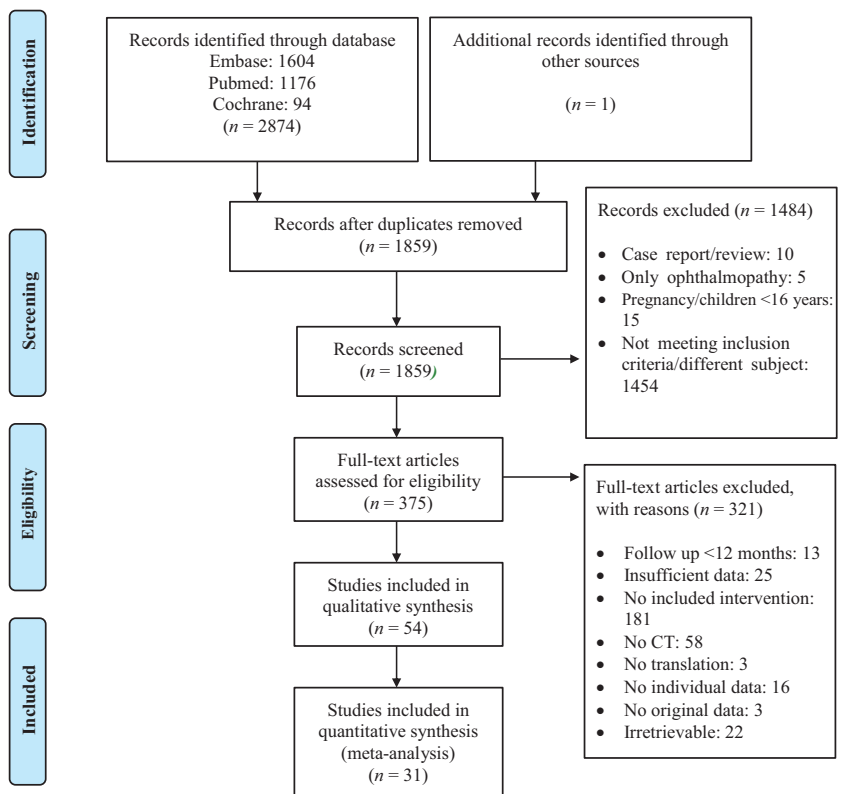

\section{Figure 1}

Study flow diagram.

\section{Data items}

From all included trials, we extracted the following data: (1) General information: first author, year of publication and country; (2) trial characteristics: design, if eligibility criteria are reported, duration, mode of randomization, blinding (assessors and patients) and intention-to-treat analysis; (3) interventions: basis intervention with ATD (days and dose) and pretreatment risk factors predicting outcome; (4) patients: number of patients included, sex and age; (5) outcomes: primary outcome, secondary outcome and effect size.

\section{Risk assessment of bias in individual studies}

All included studies were assessed by two reviewers according to the Scottish Intercollegiate Guidelines Network (SIGN) (21) (Supplementary file). Assessment was done using the appropriate checklist accordingly (i.e. RCT, cohort or case-control study). The risk of bias was judged to be unacceptable, high or low. The following aspects of internal and external validity were also assessed and reported: (1) Are there any eligibility criteria reported? (2) Mode of randomization (e.g. random number permutation table); (3) Participants blinding? (4) Assessor blinding? (5) Intention-to-treat analysis? (6) Outcome well defined and complete? (7) Mean follow-up in months; (8) Percentage of lost to follow-up?

\section{Summary measures, planned methods of analysis and risk of bias across studies}

Only risk factors reported in at least three studies were considered. Due to low number of studies, we omitted data on the following risk factors: urinary iodine, fT4I, fT3, thyroglobulin antibodies, scintigraphy, past history and family history. As none of the authors that we contacted about original data responded, we had to impute means and standard deviations for several studies as recommended, as only median and range had been reported (22). To impute a standard error for the within-study variation, Poisson distribution was assumed. To calculate thyroid volume when only a weight was given, we assumed a density of $1 \mathrm{~g}$ per $\mathrm{mL}$ (same density as water).

For dichotomous data, risk ratios with 95\% CI were calculated by a random-effects model (23). For continuous data, we computed the standardized mean difference according to Hedges (g) (24) as effect size as measurements were on different scales. To facilitate clinical interpretation, we also calculated weighted raw mean differences (RMD). As test of heterogeneity, we computed the variation in risk ratio (RR) across studies attributable to heterogeneity $\left(I^{2}\right)(25)$. Additionally, we imputed mean values for all missing data on the relapse groups in all 31 studies using a multivariate truncated linear regression model (26). To check for internal validity, we performed univariate meta-regression and compared the results to the ones obtained by classical meta-analysis. Significant results from the univariate meta-regression were fitted into a multivariate meta-regression (27) while omitting co-linear variables. Categorical data were transformed into percentages before integration into regression models. Analyses for subgroup and sensitivity analyses were planned post hoc due to the heterogeneity of studies. Gene data were assessed qualitatively, and if sufficient data were reported, we calculated the odds ratios (OR).

As most data were available for the risk factor of age, we used these to check for small study effects. For each of these trials, we plotted the effect by the inverse of its standard error (28). The symmetry of these 'funnel plots' was assessed both visually and formally with Egger's test to see if the effect decreased with increasing sample size.

The statistical analysis was conducted using Stata software version 12.1 (Stata Corp.). All significance tests were two-sided, and $P<0.05$ was considered to be statistically significant. 


\section{Results}

\section{Study selection and study characteristics}

A total of 1859 articles were evaluated by the accordingly skilled members of our study group (Fig. 1 according to suggestions of the PRISMA group (7)). Of these, 375 fulltext articles were assessed for eligibility and 54 trials were included in the final data analysis $(9,10,11,12,13,14$, $15,16,17,18,19,20,29,30,31,32,33,34,35,36,37$, $38,39,40,41,42,43,44,45,46,47,48,49,50,51,52$, $53,54,55,56,57,58,59,60,61,62,63,64,65,66,67$, $68,69,70$ ). There were 31 trials (of which 5 randomizedcontrolled and 18 controlled trials) with 2322 relapses in a total of 4346 participants (overall relapse rate $53.4 \%$ ) for quantitative meta-analysis (Supplementary Tables 1 and 2). Of note, the number of patients does not add up to the grand total as data reported were both used for the genomic data table and the quantitative analysis. Trials reporting genomic data were only evaluated qualitatively. Trials were published between 1977 and 2015. Mainly propylthiouracil and methimazole were used as ATDs.

\section{Risk of bias within studies}

Trials were mostly small with diverse risk of bias (10 high, 15 medium and 29 low risk of bias; Supplementary Table 3). Of the 31 trials included in the quantitative analysis, 12 were of low risk, 14 were of medium risk and 5 were of high risk of bias.

\section{Qualitative reporting of gene data}

Gene data were available from 13 of 54 studies (24.1\%) with a total of 2178 patients being genotyped. Number of subjects ranged from 30 to 451 per trial, and trials had a moderate-to-low risk of bias (one high, five medium and seven low risk of bias; Supplementary Table 3). Results are summarized in Table $1(9,14,20,32,35,40,41,42$, $51,54,57,60,70)$. The effect size for most markers were rather modest with OR around 1-2 in general. Many were below a size, which could be deemed clinically significant (i.e. $>2$ ), but with a wide range from 0.1 to 16.1 .

Out of 35 studies testing HLA types, only 4 studies reported their result in detail. Mostly, negative or nonsignificant associations were omitted, apparently with high risk of publication bias. De Bruin (14) showed, contradictorily to others, no increased relapse rate for DR3-positive patients.

There were also investigations on the composition of peripheral leucocytes. One study did not find a difference in peripheral T-, B- and NK-lymphocyte subsets, to sufficiently predict relapse, even not after combination of factors (66). Van Ouwerkerk (34) reported the amount of subsets of T-lymphocytes (helper and suppressor type) without any predictive value.

\section{Quantitative synthesis of results}

Random-effects meta-analysis did show a significant predictive value for orbitopathy RR 1.16 (1.08; 1.25), smoking RR 1.13 (1.02; 1.25), thyroid volume assessed by sonography g $0.54(0.18 ; 0.90) / \mathrm{RMD} 5.46 \mathrm{~mL}(1.92$; 9.00), goiter size according to WHO grades g $0.18(0.07$; $0.30)$, fT4 g $0.16(0.05 ; 0.28) / \mathrm{RMD} 3.98 \mathrm{pM}(0.53 ; 7.44)$, tT3 g $0.25(0.04 ; 0.46) /$ RMD $0.59 \mathrm{nM}(0.10 ; 1.09)$, TRAb g $0.33(0.04 ; 0.62) / \mathrm{RMD} 16.93 \mathrm{U} / \mathrm{L}(1.94 ; 31.93)$, TBII g 0.36 (0.09; 0.63)/RMD 8.27\% (2.61; 13.92), TSAb g 0.26 (0.06; $0.45) / \mathrm{RMD} 127.39 \%(18.01 ; 236.77)$, whereas male vs female sex RR 0.91 (0.82; 1.00), age g -0.14 (-0.35; 0.07)/ RMD -0.80year (-2.54; 0.94), and initial tT4 level g -0.04 $(-0.31 ; 0.23) / \mathrm{RMD} 2.11 \mathrm{nM}(-13.84 ; 18.06)$ did not show significant associations (Table 2).

As we computed standardized mean difference according to Hedges, values are comparable across different units of measurement. This gives a crude idea of effect size between different factors. A large drawback of this method is its difficult interpretation. Generally, effect sizes with $g$ values around 0.1 are viewed as small, around 0.3 as medium and above 0.5 as strong (24).

For better clinical comparison, we calculated raw mean differences. Thus, an increase of the units depicted above results in a $1 \%$ increase in relapse risk (e.g. thyroid volume $+1 \%$ relapse risk for every $5.46 \mathrm{~mL}$ increase).

Additionally, we depicted the two risk factors age and goiter stratified by the subgroups relapse vs remission for age and WHO grades for goiter (Fig. 2, additional figures in Supplementary file).

In univariable meta-regression values for TBII, tT3, fT4, sonographic thyroid volume and smoking status became non-significant, whereas tT4 turned significant. As this might be due to imputation, we refitted the models with the original data, but observed no difference.

To further check for internal validity, we refitted models with values for remission. These models showed similar results except for the gender of participants were there was a collinear increase of remission and relapse with both sexes. This might be explained by the low statistical power of meta-regression and the small effect of sex on relapse risk. Also, age and tT3 showed contradictory effect sizes, which we interpreted were due to small effect 
Table 1 Associated genes for relapse.

\begin{tabular}{|c|c|c|c|c|c|}
\hline Gene locus & $\begin{array}{l}\text { SNP polymorphism of } \\
\text { gene and location }\end{array}$ & $\begin{array}{l}\text { Receptor or } \\
\text { transcript }\end{array}$ & Allele & $\begin{array}{l}\text { Effect size }(95 \% \\
\text { confidence interval) } \\
\text { for risk of relapse }\end{array}$ & Reported by \\
\hline \multirow[t]{2}{*}{ Ch $6 p 21.31$} & N/A & HLA-DR3 & N/A & $\begin{array}{l}\text { RR } 0.29(P 0.016) / \\
\quad \text { OR } 0.1(0.0 ; 0.7)\end{array}$ & $\begin{array}{l}\text { de Bruin (1988), } \\
\text { The Netherlands }\end{array}$ \\
\hline & & HLA-Cw7 & & $\begin{array}{l}\text { RR } 2.05(P 0.009) / \\
\quad \text { OR } 5.0(1.4 ; 17.7)\end{array}$ & \\
\hline \multirow[t]{3}{*}{ Ch $6 p 21.31$} & N/A & HLA-A1 & $\mathrm{N} / \mathrm{A}$ & OR $0.5(0.2 ; 1.3)$ & Allanic (1983), France \\
\hline & & HLA-B8 & & OR $1.1(0.4 ; 3.1)$ & \\
\hline & & HLA-DR3 & & OR $1.8(0.6 ; 4.9)$ & \\
\hline \multirow[t]{2}{*}{ Ch $6 p 21.31$} & $\mathrm{~N} / \mathrm{A}$ & HLA-A1 & $\mathrm{N} / \mathrm{A}$ & OR $2.2(0.8 ; 6.1)$ & Irvine (1977), UK \\
\hline & & HLA-B8 & & OR $3.3(1.2 ; 9.6)$ & \\
\hline \multirow[t]{3}{*}{ Ch $6 p 21.31$} & N/A & HLA-B12 & $\mathrm{N} / \mathrm{A}$ & $\begin{array}{l}\text { RR } 0.23(P 0.02) / O R \\
0.2(0.0 ; 1.1)\end{array}$ & $\begin{array}{l}\text { Schifferdecker (1991), } \\
\text { Germany }\end{array}$ \\
\hline & & HLA-DR5 & & $\begin{array}{l}\text { RR } 9.85(P 0.03) / \mathrm{OR} \\
9.9(1.2 ; 438.0)\end{array}$ & \\
\hline & & HLA-DR7 & & $\begin{array}{l}\text { RR } 0.27(P 0.05) / O R \\
\quad 0.3(0.1 ; 1.3)\end{array}$ & \\
\hline Ch $6 p 21.31$ & $\mathrm{~N} / \mathrm{A}$ & HLA-DR3 & N/A & OR $1.2(0.7 ; 2.4)$ & $\begin{array}{l}\text { Schleusener (1987), } \\
\text { Germany }\end{array}$ \\
\hline Ch $6 p 21.31$ & $\mathrm{~N} / \mathrm{A}$ & HLA-DR3 & $\mathrm{N} / \mathrm{A}$ & OR $0.8(0.5 ; 1.4)$ & $\begin{array}{l}\text { Schleusener (1989), } \\
\text { Germany }\end{array}$ \\
\hline Ch 6p21.31 & N/A & HLA-DR3 & N/A & OR $2.2(0.4 ; 12.3)$ & Wilson (1986), Scotland \\
\hline \multirow[t]{3}{*}{ Ch $20 q 12-q 13.2$} & 5' UTR & CD40 & $\mathrm{CC}$ & OR $1.2(0.5 ; 2.9)$ & Kim (2007), South Korea \\
\hline & & & $\mathrm{CT}$ & OR $0.6(0.3 ; 1.4)$ & \\
\hline & & & $\mathrm{TT}$ & OR $3.7(0.5 ; 166.2)$ & \\
\hline \multirow[t]{3}{*}{ Ch $20 q 12-q 13.2$} & Intron 1 (rs745307) & CD 40 & $\mathrm{C} / \mathrm{T}$ vs $\mathrm{C} / \mathrm{C}$ & OR $8.0(1.0 ; 62.7)$ & Wang (2013), Taiwan \\
\hline & Intron 1 (rs11569309) & & $\mathrm{C} / \mathrm{T}$ vs $\mathrm{T} / \mathrm{T}$ & OR $8.1(1.0 ; 63.7)$ & \\
\hline & Intron 8 (rs3765457) & & $A / G$ vs $A / A$ & OR $2.6(1.1 ; 6.3)$ & \\
\hline Ch 2 p33 & $\begin{array}{l}\text { Position +49A/G in } \\
\text { exon } 1 \text { (rs231775) }\end{array}$ & CTLA4 & $\begin{array}{r}\mathrm{G} / \mathrm{G} \text { vs } \mathrm{A} / \mathrm{G} \\
\text { and } \mathrm{A} / \mathrm{A}\end{array}$ & OR $2.0(1.2 ; 3.3)$ & Wang (2013), Taiwan \\
\hline \multirow[t]{3}{*}{ Ch $2 p 33$} & CT60 polymorphism & CTLA4 & AA & OR $0.7(0.0 ; 42.2)$ & Kim (2007), South Korea \\
\hline & in $3^{\prime}$ UTR & & AG & OR $1.1(0.4 ; 3.5)$ & \\
\hline & & & GG & OR $0.9(0.3 ; 2.5)$ & \\
\hline \multirow[t]{3}{*}{ Ch $2 p 33$} & Promoter at position & CTLA4 & $\mathrm{CC}$ & OR $1.1(0.4 ; 2.7)$ & Kim (2007), South Korea \\
\hline & -318 & & $\mathrm{CT}$ & OR $0.9(0.4 ; 2.2)$ & \\
\hline & & & TT & OR $0.4(0.0 ; 31.9)$ & \\
\hline \multirow[t]{3}{*}{ Ch 2p33 } & Position +49 in & CTLA4 & AA & OR $1.9(0.2 ; 96.2)$ & Kim (2007), South Korea \\
\hline & exon 1 & & AG & OR $1.5(0.4 ; 5.5)$ & \\
\hline & & & GG & OR $0.5(0.1 ; 1.6)$ & \\
\hline \multirow[t]{3}{*}{ Ch $2 p 33$} & Position +49 in & CTLA4 & AA & OR $1.2(0.2 ; 5.2)$ & Wang (2004), Taiwan \\
\hline & exon 1 & & AG & OR $0.3(0.1 ; 0.7)$ & \\
\hline & & & GG & OR $4.0(1.9 ; 8.6)$ & \\
\hline \multirow[t]{3}{*}{ Ch 2p33 } & Position 49 in exon 1 & CTLA4 & AA & OR $0.3(0.1 ; 0.8)$ & Tanrikulu (2006), Turkey \\
\hline & & & AG & OR $6.2(2.4 ; 16.6)$ & \\
\hline & & & GG & OR $3.6(0.8 ; 5.7)$ & \\
\hline \multirow[t]{3}{*}{ Ch $8 q 24$} & Exon 33 & Thyroglobulin & $\mathrm{CC}$ & OR $1.5(0.6 ; 3.6)$ & Tanrikulu (2006), Turkey \\
\hline & & & $\mathrm{CT}$ & OR $1.4(0.6 ; 3.4)$ & \\
\hline & & & $\mathrm{TT}$ & OR $2.1(0.7 ; 6.5)$ & \\
\hline \multirow[t]{3}{*}{ Ch $20 q 13.2-q 13.3$} & T393C polymorphism & G alpha subunit of & $\mathrm{CC}$ & OR $1.3(0.7 ; 2.7)$ & Glowacka (2009), \\
\hline & & G protein & $\mathrm{CT}$ & OR $0.5(0.3 ; 0.9)$ & Germany \\
\hline & & (GNAS1) & TT & OR $2.4(1.0 ; 6.2)$ & \\
\hline \multirow[t]{3}{*}{ Ch 9p24.1 } & Position 8923 in & Programmed cell & AA & OR $0.4(0.1 ; 1.4)$ & Hayashi (2008), Japan \\
\hline & PD-L1 intron 4 & death-1 ligand & $A C$ & OR $1.5(0.3 ; 5.0)$ & \\
\hline & & & CC & OR $16.1(0.8 ; 954.2)$ & \\
\hline
\end{tabular}




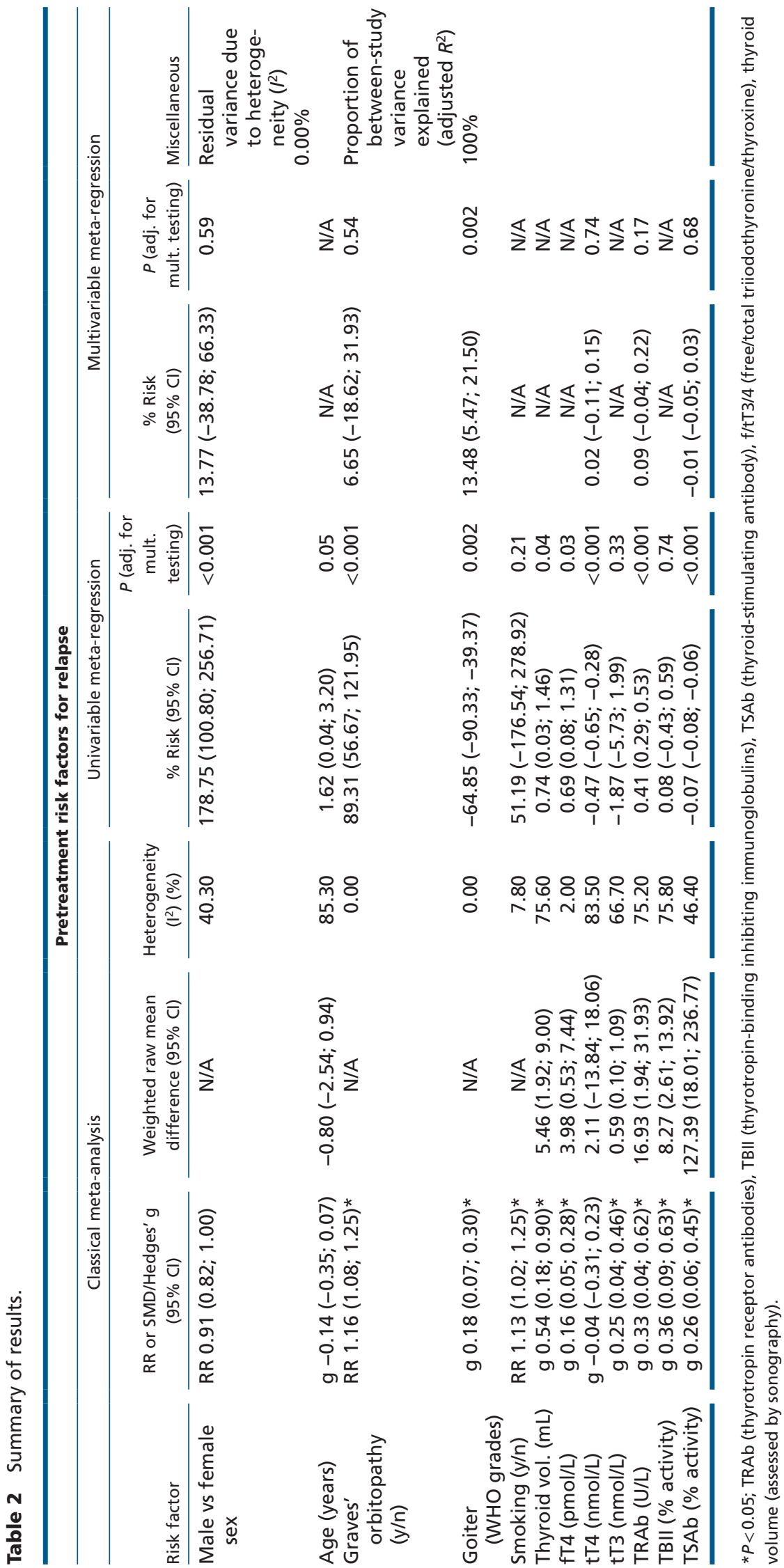


A

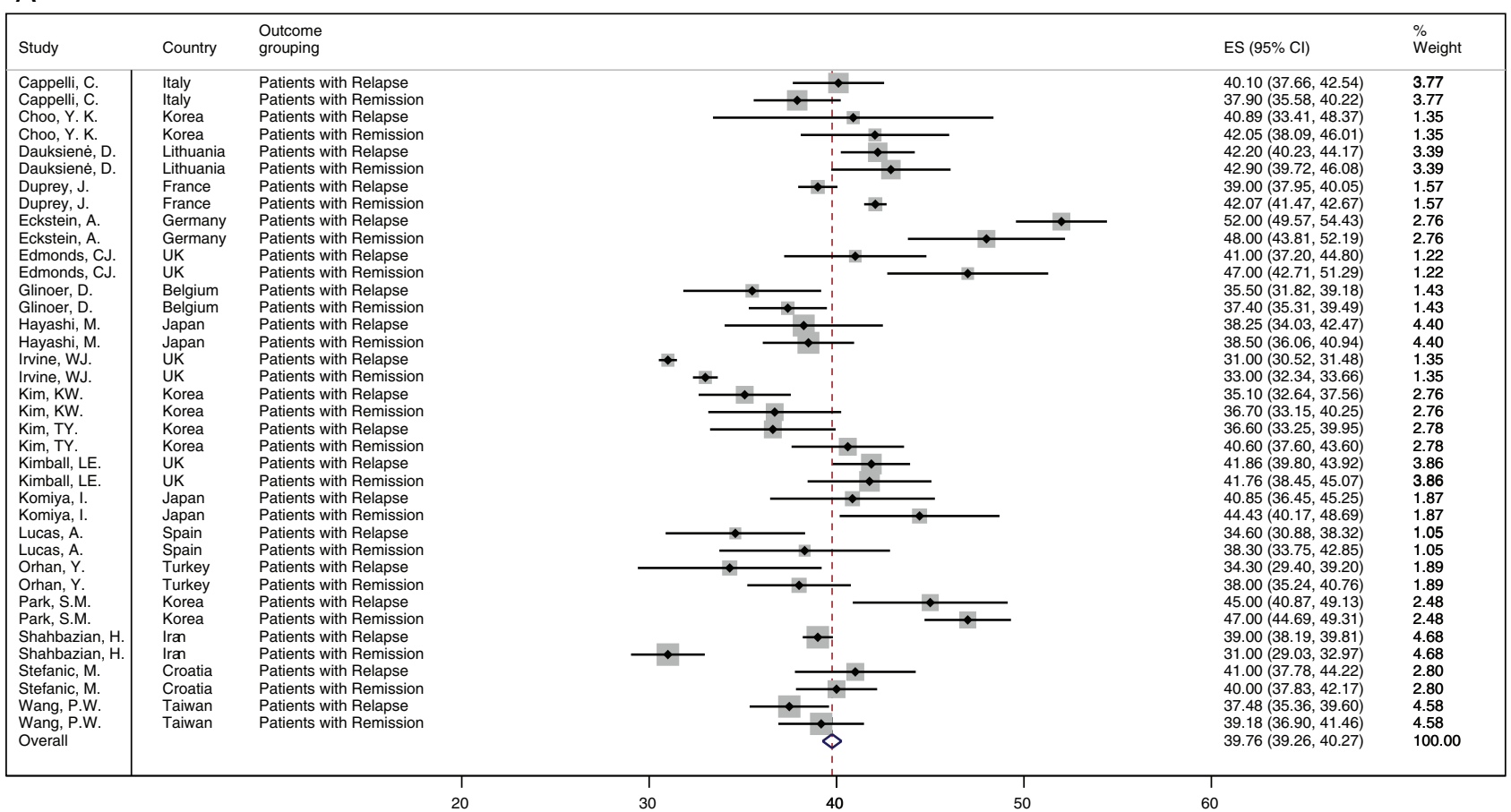

B

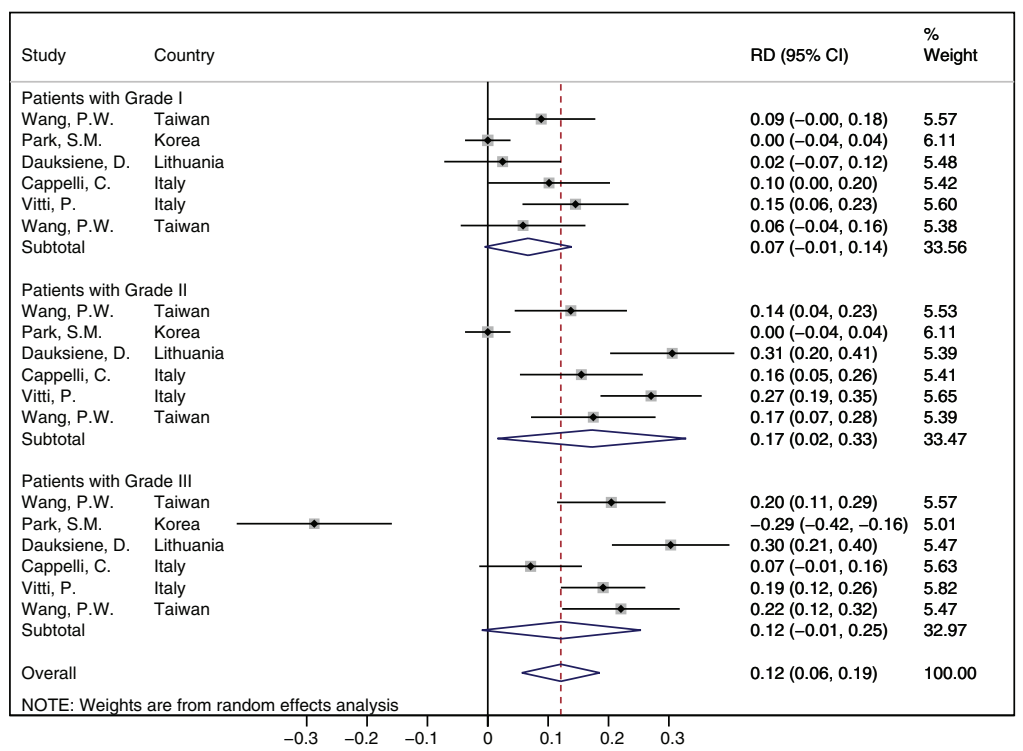

\section{Figure 2}

(A) Relapse risk according to age at diagnosis. (B) Relapse risk according to WHO goiter grade.

size and scarcity of data respectively. These results and measurement of heterogeneity by $I^{2}$ fitted well with the classical meta-analysis, except the ones noted previously.

In multivariate meta-regression, we first fitted a model with all significant covariates from univariate regression. As these results could be influenced by imputation, we refitted the models with the original data, but observed no difference again. Therefore, we concluded that influential, biased studies attributed to these observed effects.

As data were handled as percentages, Table 2 shows a percentage increase or decrease for every unit of risk factor respectively. As an example, it can be depicted that a male with Graves' orbitopathy and goiter grade II has a $47 \%$ increased risk for relapse (cumulative 
risk $=14 \%+7 \%+(2 \times 13 \%)=47 \%)$ compared to a female patient without orbitopathy and no goiter.

\section{Risk of bias across studies}

As there was strong evidence for heterogeneity for most risk factors, we computed a funnel plot (Supplementary Fig. 2) for age as a risk factor showing slight asymmetry. We chose age as most data were available on this risk factor. Egger's test for no small study effects did show a significant result, thus rejecting publication bias across studies $(P=0.006)$. Of course, these results may also be influenced by small study effects, poor design of the included studies and true heterogeneity considering the genetic differences in the studied populations (28).

\section{Discussion}

\section{Summary of evidence}

This is the first systematic review and meta-analysis focusing on pretreatment risk factors predicting relapse before the first treatment course with ATD in patients with hyperthyroidism due to Graves' disease. The 54 included trials had moderate-to-low trial quality and risk of bias. The pooled analysis showed a good prediction of relapse with several risk factors, but in most trials, these factors by themselves had no enough power. Risk factors were similar in randomized trials compared to case-controlled and controlled trials.

Graves' disease is an autoimmune disease caused by the stimulation of antibodies directed against the thyrotropin receptor in most patients (4). The risk for disease occurrence depends by part on environmental factors such as smoking and psychological factors such as stressful life events, and by part on genetic factors as previously demonstrated in twin studies $(4,71)$. Although environmental factors for relapse could be altered, genetic factors could not be directly influenced. But it would be possible to modify the immune system's reaction by immunosuppressive drugs (72) until the environmental co-factors subside.

Our analysis has two main clinical benefits. First, it offers clinicians an up-to-date qualitative overview of genetic factors and other risk factor associated with the recurrence of Graves' disease. Second, the quantitative analysis also allows to calculate the risk for relapse in an individual patient. Such numbers may be used when discussing the therapeutic options with patients, i.e. whether medical therapy or more definite treatment options (radio-iodine and surgery) should be used. Still, the effect sizes for most risk factors assessed in our analysis were small and statistically non-significant. Thus, calculation of relapse risk based on this analysis should be used with caution and only in conjunction with good clinical judgment. Also, a prospective study should validate our findings before more widespread use in clinical practice.

\section{Strengths and limitations}

The strengths of our study are the predefined study protocol adhering to PRISMA guidelines for systematic reviews, the inclusion from studies all around the globe without language restrictions and thus reflecting different ethnicities and genetic backgrounds (2) and the relevance of this topic in every day practice. As one might argue that the response to ATD depends on genetic background, our robust findings of the included trials from different countries suggest otherwise. This increases the clinical applicability and external validity. Although we do not provide insights into new risk factor and there have already been various systematic reviews assessing the different treatment options for Graves' disease $(1,5,6,73)$, we provide the very first systematic review on pretreatment risk factors so far.

We are aware of several limitations in regard to this analysis. First, retrieved data were very heterogeneous and authors of eligible studies could either not be contacted or refused to share their date with us. Thus, we had to rely on several imputation methods, which could have influenced our results. For our data set, we assumed that data were missing at random. A complete-case analysis would introduce bias and lower the power of the analysis. By using multiple imputation, we assume that bias is minimal as the approach is generally considered to be conservative. Still, imputation has limitations particularly if data are not normally distributed.

Secondly, trials were mostly monocentric, heterogeneous and a lot had a rather high risk for bias. Many of the included studies were not randomized or case-controlled trials. Thirdly, there were many different laboratory assays over a wide time span of 38 years, making comparability difficult, especially for the immunological antibody assays. In addition, only two studies had blinding of participants or assessors (placebo controlled). No trial had an intention-to-treat analysis performed and adverse effects were rarely, if at all, systematically reported. Still, studies reported eligibility criteria, and mean follow-up was at least one year after the withdrawal of ATD, mainly 24 months. Notably, most relapses occur 
within the first two years. Still, studies reported eligibility criteria and mean follow-up was at least one year after the withdrawal of ATD, mainly 24 months. Heterogeneity of data did not allow for formal quantitative analysis (e.g. mean time to relapse). Hence, we qualitatively present these data without formally analyzing it (Supplementary Table 2). Roughly $2 / 3$ of the relapses occurred between months 6 and 18 after stopping ATD, being in line with current literature (1).

Meta-regression is prone to over-interpretation of findings. However, in our case, results were similar to classical meta-analysis. Furthermore, by adjusting $P$ values for multiple testing, we are certain that the results reflect true findings.

Overall, the markers identified by our systematic review, either alone or combined, were not strong enough to predict the clinical outcome of a single patient. Ideally, fresh markers would fill this gap. In this regard, a very recent publication from the Netherlands might shed new light. The authors generated a risk score based on a multivariate analysis of 173 prospectively enrolled patients subjected to their first ATD course. They identified lower age, higher fT4, higher TBII, large goiters, PTPN22 C/T SNP and HLA types DQB1*02, DQA $1{ }^{*} 05$ and DRB $1 * 03$ as independent risk factors (74). Still, these results need to be externally validated and replicated in a larger cohort first. However, this approach would lead to a new perspective and could be the first step to a form of individualized treatment in Graves' disease.

\section{Conclusions}

In conclusion, this systematic review and metaanalysis found several pretreatment risk factors of relapse in patients with standard treatment of Graves' disease. The occurrence of orbitopathy, smoking, larger thyroid volume and biochemically more severe disease (fT4, tT3, TRAb, TBII and TSAb levels) did show a higher risk of relapse.

Yet, the small predictive power of a single risk factor is insufficient to predict the outcome of a single patient. This calls for novel markers to be identified and tested in future trials.

Supplementary data

This is linked to the online version of the paper at http://dx.doi.org/10.1530/ EJE-16-0725.

\section{Declaration of interest}

The authors declare that there is no conflict of interest that could be perceived as prejudicing the impartiality of the research reported.

\section{Funding}

This study was supported in part by the Swiss National Science Foundation (SNSF Professorship, PP00P3_150531/1) and the Research Council of the Kantonsspital Aarau (1410.000.044). Funders had no role in the design, analysis or writing of this article.

\section{Author contribution statement}

T S, B M and P S designed the study and H F and C D analyzed the data. $\mathrm{H} \mathrm{F}$ and T S wrote the first draft of the manuscript and had primary responsibility for the final content. All authors read and approved the final manuscript.

\section{Acknowledgments}

The authors want to express their gratitude to Alexander Litke, MD, and Merih Guglielmetti for their translation of Russian and Turkish articles respectively.

\section{References}

1 Genovese BM, Noureldine SI, Gleeson EM, Tufano RP \& Kandil E. What is the best definitive treatment for Graves' disease? A systematic review of the existing literature. Annals of Surgical Oncology 201320 660-667. (doi:10.1245/s10434-012-2606-x)

2 Antonelli A, Ferrari SM, Corrado A, Di Domenicantonio A \& Fallahi P. Autoimmune thyroid disorders. Autoimmunity Reviews 201514 174-180. (doi:10.1016/j.autrev.2014.10.016)

3 Orgiazzi J. Thyroid autoimmunity. Presse Médicale 20124125. (doi:10.1016/j.lpm.2012.10.002)

4 Franklyn JA \& Boelaert K. Thyrotoxicosis. Lancet 2012379 1155-1166. (doi:10.1016/S0140-6736(11)60782-4)

5 Sundaresh V, Brito JP, Wang Z, Prokop LJ, Stan MN, Murad MH \& Bahn RS. Comparative effectiveness of therapies for Graves' hyperthyroidism: a systematic review and network meta-analysis. Journal of Clinical Endocrinology and Metabolism 201398 3671-3677. (doi:10.1210/jc.2013-1954)

6 Abraham P, Avenell A, McGeoch SC, Clark LF \& Bevan JS. Antithyroid drug regimen for treating Graves' hyperthyroidism. Cochrane Database of Systematic Reviews 2010 CD003420. (doi:10.1002/14651858. CD003420.pub4)

7 Liberati A, Altman DG, Tetzlaff J, Mulrow C, Gøtzsche PC, Ioannidis JPA, Clarke M, Devereaux PJ, Kleijnen J \& Moher D. The PRISMA statement for reporting systematic reviews and meta-analyses of studies that evaluate health care interventions: explanation and elaboration. PLoS Medicine 20096 e1000100. (doi:10.1371/journal.pmed.1000100)

8 Higgins JPT \& Green S. Cochrane Handbook for Systematic Reviews of Interventions, Version 5.1.0, updated 2011. (available at: http:// handbook.cochrane.org/). Accessed on 16 October 2015.

9 Wang PW, Liu RT, Juo SHH, Wang ST, Hu YH, Hsieh CJ, Chen MH, Chen IY \& Wu CL. Cytotoxic T lymphocyte-associated molecule-4 polymorphism and relapse of Graves' hyperthyroidism after antithyroid withdrawal. Journal of Clinical Endocrinology and Metabolism 200489 169-173. (doi:10.1210/jc.2003-030854)

10 Orunesu E, Bagnasco M, Salmaso C, Altrinetti V, Bernasconi D, Del Monte P, Pesce G, Marugo M \& Mela GS. Use of an artificial neural network to predict Graves' disease outcome within 2 years of drug withdrawal. European Journal of Clinical Investigation 200434 210-217. (doi:10.1111/j.1365-2362.2004.01318.x)

11 Sonnet E, Massart C, Gibassier J, Allannic H \& Maugendre D. Longitudinal study of soluble intercellular adhesion molecule-1 (ICAM1) in sera of patients with Graves' disease. Journal of Endocrinological Investigation 199922 430-435. (doi:10.1007/bf03343586) 
12 Vitti P, Rago T, Chiovato L, Pallini S, Santini F, Fiore E, Rocchi R, Martino E \& Pinchera A. Clinical features of patients with Graves disease undergoing remission after antithyroid drug treatment. Thyroid 19977 369-375. (doi:10.1089/thy.1997.7.369)

13 Massart C, Guilhem I, Gibassier J, Allannic H \& Nicol M. Comparison of thyroperoxidase and microsomal antibody assays in sera from patients with Graves disease. Clinical Chemistry 199137 1777-1780.

14 de Bruin TW, Bolk JH, Bussemaker JK, Stijnen T, Schreuder GM, de Vries RR \& van der Heide D. Graves' disease: immunological and immunogenetic indicators of relapse. British Medical Journal (Clinical Research Edition) 1988296 1292-1295. (doi:10.1136/bmj.296.6632.1292)

15 Kimball LE, Kulinskaya E, Brown B, Johnston C \& Farid NR. Does smoking increase relapse rates in Graves' disease? Journal of Endocrinological Investigation 200225 152-157. (doi:10.1007/bf03343979)

16 Laurberg P, Buchholtz Hansen PE \& Iversen E. Goitre size and outcome of medical treatment of Graves' disease. Acta Endocrinologica 1986111 39-43. (doi:10.1530/acta.0.1110039)

17 Takaichi Y, Tamai H, Honda K, Nagai K, Kuma K \& Nakagawa T. The significance of antithyroglobulin and antithyroidal microsomal antibodies in patients with hyperthyroidism due to Graves' disease treated with antithyroidal drugs. Journal of Clinical Endocrinology and Metabolism 198968 1097-1100. (doi:10.1210/jcem-68-6-1097)

18 Yamada T, Aizawa T, Koizumi Y, Komiya I, Ichikawa K \& Hashizume K. Age-related therapeutic response to antithyroid drug in patients with hyperthyroid Graves' disease. Journal of the American Geriatrics Society 199442 513-516. (doi:10.1111/j.1532-5415.1994.tb04973.x)

19 Khanna CM, Shankar LR, Jaggi CB, Bansal JK \& Chugh P. Predictor of outcome of hyperthyroidism due to Graves disease: serum triiodothyronine/thyroxine ratio. Journal of the Association of Physicians of India 199644 98-101.

20 Wilson R, McKillop JH \& Henderson N. The ability of the serum thyrotrophin receptor antibody (TRAb) index and HLA status to predict long-term remission of thyrotoxicosis following medical therapy for Graves's disease. Clinical Endocrinology 198625 151-156. (doi:10.1111/j.1365-2265.1986.tb01676.x)

21 Scottish Intercollegiate Guidelines Network (SIGN), 2015. (available at: http://www.sign.ac.uk/index.html). Accessed on 16 October 2015.

22 Leon-Guerrero A \& Frankfort-Nachmias C. Essentials of Social Statistics for a Diverse Society. Thousand Oaks, CA, USA: SAGE, 2012.

23 DerSimonian R \& Laird N. Meta-analysis in clinical trials revisited. Contemporary Clinical Trials 201545 (Part A) 139-145. (doi:10.1016/j. cct.2015.09.002)

24 Borenstein M, Hedges LV, Higgins JPT \& Rothstein HR. Introduction to Meta-Analysis. Chichester, UK: Wiley, 2010.

25 Higgins JPT, Thompson SG, Deeks JJ \& Altman DG. Measuring inconsistency in meta-analyses. BMJ 2003327 557-560. (doi:10.1136/ bmj.327.7414.557)

26 Raghunathan TE, Lepkowski JM, van Hoewyk J \& Solenberger P. A multivariate technique for multiply imputing missing values using a sequence of regression models. Survey Methodology 200127 85-96.

27 Thompson SG \& Higgins JPT. How should meta-regression analyses be undertaken and interpreted? Statistics in Medicine 200221 1559-1573. (doi:10.1002/sim.1187)

28 Egger M, Davey Smith G, Schneider M \& Minder C. Bias in metaanalysis detected by a simple, graphical test. BMJ 1997315 629-634. (doi:10.1136/bmj.315.7109.629)

29 Yamada T, Komiya I, Miyahara Y, Komatsu M, Shima I, Inazawa T \& Aizawa T. Effect of methimazole treatment for 2 years on circulating IL-4, IgE, TBII, and TSAb in patients with hyperthyroid Graves' disease. Endocrine Journal 200653 783-788. (doi:10.1507/endocrj. K06-054)

30 Wilson R, McKillop JH \& Pearson DWM. Relapse of Graves' disease after medical therapy: predictive value of thyroidal technetium-99m uptake and serum thyroid stimulating hormone receptor antibody levels. Journal of Nuclear Medicine 198526 1024-1028.
31 Wang PW, Liu RT, Tung SC, Chien WY, Lu YC, Chen CH, Kuo MC, Hsieh JR \& Wang ST. Outcome of Graves' disease after antithyroid drug treatment in Taiwan. Journal of the Formosan Medical Association 199897 619-625.

32 Wang PW, Chen IY, Juo SH, Hsi E, Liu RT \& Hsieh CJ. Genotype and phenotype predictors of relapse of graves' disease after antithyroid drug withdrawal. European Thyroid Journal 20131 251-258. (doi:10.1159/000342621)

33 Vitti P, Rago T, Mancusi F, Pallini S, Tonacchera M, Santini F, Chiovato L, Marcocci C \& Pinchera A. Thyroid hypoechogenic pattern at ultrasonography as a tool for predicting recurrence of hyperthyroidism after medical treatment in patients with Graves' disease. Acta Endocrinologica 1992126 128-131.

34 van Ouwerkerk BM, Krenning EP \& Docter R. Cellular and humoral immunity in patients with hyperthyroid Graves' disease before, during and after antithyroid drug treatment. Clinical Endocrinology 198726 385-394. (doi:10.1111/j.1365-2265.1987.tb00795.x)

35 Tanrikulu S, Erbil Y, Ademoglu E, Issever H, Barbaros U, Kutluturk F, Ozarmagan S \& Tezelman S. The predictive value of CTLA-4 and Tg polymorphisms in the recurrence of Graves' disease after antithyroid withdrawal. Endocrine 200630 377-381. (doi:10.1007/s12020-0060017-0)

36 Takamatsu J, Kuma K \& Mozai T. Serum triiodothyronine to thyroxine ratio: a newly recognized predictor of the outcome of hyperthyroidism due to Graves' disease. Journal of Clinical Endocrinology and Metabolism 198662 980-983. (doi:10.1210/jcem-62-5-980)

37 Stefanic M \& Karner I. Thyroid peroxidase autoantibodies are associated with a lesser likelihood of late reversion to hyperthyroidism after successful non-ablative treatment of Graves' disease in Croatian patients. Journal of Endocrinological Investigation 201437 71-77. (doi:10.1007/s40618-013-0026-9)

38 Soveid M, Shaabani A, Ghaedi Gh H, Jafari SM \& Omrani G. Prognostic factors in the relapse of Graves' disease following treatment with antithyroid drugs. Iranian Journal of Medical Sciences 200328 106-110.

39 Shahbazian H, Saiedinia S \& Aleali AM. Outcome of graves disease after anti-thyroid drug treatment in south west of Iran. Pakistan Journal of Medical Sciences 201228 445-449.

40 Schleusener H, Schwander J, Fischer C, Holle R, Holl G, Badenhoop K, Hensen J, Finke R, Bogner U, Mayr WR et al. Prospective multicentre study on the prediction of relapse after antithyroid drug treatment in patients with Graves' disease. Acta Endocrinologica 1989120 689-701. (doi:10.1530/acta.0.1200689)

41 Schleusener H, Schwander J, Holl G, Badenhoop K, Hensen J, Finke R, Schernthaner G, Mayr WR \& Kotulla P. Do HLA-DR-typing and measurement of TSH-receptor antibodies help in the prediction of the clinical course of Graves' thyrotoxicosis after antithyroid drug treatment? Acta Endocrinologica: Supplementum 1987281 318-324.

42 Schifferdecker E, Kuhnl P, Schoffling K, Manfras B, Holzberger G, Spielmann W \& Bohm BO. Immunogenetic markers in patients with Graves' disease. Klinische Wochenschrift 199169 256-260. (doi:10.1007/BF01666851)

43 Scazziga BR. Drug therapy of Basedow's disease. Long term functional results (302 cases). Schweizerische Medizinische Wochenschrift 1977107 423-427.

44 Romaldini JH, Bromberg N, Werner RS, Tanaka LM, Rodrigues HF, Werner MC, Farah CS \& Reis LC. Comparison of effects of high and low dosage regimens of antithyroid drugs in the management of Graves' hyperthyroidism. Journal of Clinical Endocrinology and Metabolism 198357 563-570. (doi:10.1210/jcem-57-3-563)

45 Prakash R. Prediction of remission in Graves' disease treated with long-term carbimazole therapy: evaluation of technetium-99m thyroid uptake and TSH concentrations as prognostic indicators. European Journal of Nuclear Medicine 199623 118-122. (doi:10.1007/ BF01731833) 
46 Park SM, Cho YY, Joung JY, Sohn SY, Kim SW \& Chung JH. Excessive iodine intake does not increase the recurrence rate of Graves' disease after withdrawal of the antithyroid drug in an iodine-replete area. European Thyroid Journal 20154 36-42. (doi:10.1159/000375261)

47 Orhan Y, Azezli A, Aral F, Tascioglu C, Molvalilar S \& Sencer E. The frequency of remission and relapse in Graves' disease was investigated. Istanbul Üniversitesi Tıp Fakültesi Mecmuası 199255 525-530.

48 Murakami M, Koizumi Y, Aizawa T, Yamada T, Takahashi Y, Watanabe T \& Kamoi K. Studies of thyroid function and immune parameters in patients with hyperthyroid Graves' disease in remission. Journal of Clinical Endocrinology and Metabolism 198866 103-108. (doi:10.1210/jcem-66-1-103)

49 Lucas A, Salinas I, Rius F, Pizarro E, Granada ML, Foz M \& Sanmarti A. Medical therapy of Graves' disease: does thyroxine prevent recurrence of hyperthyroidism? Journal of Clinical Endocrinology and Metabolism 199782 2410-2413. (doi:10.1210/jcem.82.8.4118)

50 Komiya I, Yamada T, Sato A, Kouki T, Nishimori T \& Takasu N. Remission and recurrence of hyperthyroid Graves' disease during and after methimazole treatment when assessed by IgE and interleukin 13. Journal of Clinical Endocrinology and Metabolism 200186 3540-3544. (doi:10.1210/jcem.86.8.7734)

51 Kim KW, Park YJ, Kim TY, Park DJ, Park KS \& Cho BY. Susceptible alleles of the CD40 and CTLA-4 genes are not associated with the relapse after antithyroid withdrawal in Graves' disease. Thyroid 2007 17 1229-1234. (doi:10.1089/thy.2007.0011)

52 Kim TY, Park YJ, Park DJ, Chung H-K, Kim WB, Kohn LD \& Cho BY. Epitope heterogeneity of thyroid-stimulating antibodies predicts long-term outcome in Graves' patients treated with antithyroid drugs. Journal of Clinical Endocrinology and Metabolism 200388 117-124. (doi:10.1210/jc.2002-020389)

53 Jonas M, Ambroziak U, Bednarczuk T \& Nauman J. Predicting a relapse of Graves' hyperthyroidism in adults during the early phase of treatment with anti-thyroid drugs. Endokrynologia Polska 200657 596-604.

54 Irvine WJ, Gray RS, Morris PJ \& Ting A. Correlation of HLA and thyroid antibodies with clinical course of thyrotoxicosis treated with antithyroid drugs. Lancet 19772 898-900. (doi:10.1016/S01406736(77)90833-9)

55 Hou X, Li Y, Li J, Wang W, Fan C, Wang H, Zhang H, Shan Z \& Teng W. Development of thyroid dysfunction and autoantibodies in Graves' multiplex families: an eight-year follow-up study in Chinese Han pedigrees. Thyroid 201121 1353-1358. (doi:10.1089/thy.2011.0035)

56 Hoermann R, Quadbeck B, Roggenbuck U, Szabolcs I, Pfeilschifter J, Meng W, Reschke K, Hackenberg K, Dettmann J, Prehn B et al. Relapse of Graves' disease after successful outcome of antithyroid drug therapy: Results of a prospective randomized study on the use of levothyroxine. Thyroid 200212 1119-1128. (doi:10.1089/105072502321085225)

57 Hayashi M, Kouki T, Takasu N, Sunagawa S \& Komiya I. Association of an A/C single nucleotide polymorphism in programmed cell deathligand 1 gene with Graves' disease in Japanese patients. European Journal of Endocrinology 2008158 817-822. (doi:10.1530/EJE-07-0649)

58 Hansen PEB. The recurrence-rate following thyrostatic treatment of patients with hyperthyroidism and diffuse goitre (Graves' disease). The relationship to the concentrations of inorganic iodine in the plasma. Ugeskrift for Laeger 1983145 980-982.

59 Guilhem I, Massart C, Poirier JY \& Maugendre D. Differential evolution of thyroid peroxidase and thyrotropin receptor antibodies in Graves' disease: thyroid peroxidase antibody activity reverts to pretreatment level after carbimazole withdrawal. Thyroid $2006 \mathbf{1 6}$ 1041-1045. (doi:10.1089/thy.2006.16.1041)

60 Glowacka D, Loesch C, Johnson KT, Mann K, Esser J, Morgenthaler NG, Siffert W, Schmid KW \& Eckstein AK. The T393C polymorphism of the Galphas gene (GNAS1) is associated with the course of Graves' disease. Hormone and Metabolic Research 200941 430-435. (doi:10.1055/s-0029-1220902)
61 Glinoer D, Nayer P de \& Bex M. Effects of 1-thyroxine administration, TSH-receptor antibodies and smoking on the risk of recurrence in Graves' hyperthyroidism treated with antithyroid drugs: a double-blind prospective randomized study. European Journal of Endocrinology/European Federation of Endocrine Societies 2001144 475-483. (doi:10.1530/eje.0.1440475)

62 Edmonds CJ \& Tellez M. Treatment of Graves' disease by carbimazole: high dose with thyroxine compared to titration dose. European Journal of Endocrinology/European Federation of Endocrine Societies 1994131 120-124. (doi:10.1530/eje.0.1310120)

63 Eckstein AK, Lax H, Losch C, Glowacka D, Plicht M, Mann K, Esser J \& Morgenthaler NG. Patients with severe Graves' ophthalmopathy have a higher risk of relapsing hyperthyroidism and are unlikely to remain in remission. Clinical Endocrinology 200767 607-612. (doi:10.1111/j.1365-2265.2007.02933.x)

64 Duprey J \& Colliard M. Results of treatment of Graves' disease by antithyroid drugs. Report of 90 cases (author's transl). Annales d'endocrinologie 198142 233-249.

65 Dauksiene D, Dauksa A \& Mickuviene N. Independent pretreatment predictors of Graves' disease outcome. Medicina 201349 427-434.

66 Corrales JJ, Lopez A, Ciudad J \& Orfao A. The distribution of the major peripheral blood T, B and NK cell subsets does not predict the clinical outcome of Graves' disease patients after methimazole therapy. Journal of Biological Regulators and Homeostatic Agents 200114 193-199.

67 Choo YK, Yoo WS, Kim DW \& Chung HK. Hypothyroidism during antithyroid drug treatment with methimazole is a favorable prognostic indicator in patients with Graves' disease. Thyroid 201020 949-954. (doi:10.1089/thy.2009.0126)

68 Cappelli C, Gandossi E, Castellano M, Pizzocaro C, Agosti B, Delbarba A, Pirola I, Martino E de \& Rosei EA. Prognostic value of thyrotropin receptor antibodies (TRAb) in Graves' disease: a 120 months prospective study. Endocrine Journal 200754 713-720. (doi:10.1507/endocrj.K06-069)

69 Bo Youn C, Min Ho S, Ka Hee Y, Hong Kyo L, Chang-Soon K \& Hun Ki M. Evaluation of serum basal thyrotrophin levels and thyrotrophin receptor antibody activities as prognostic markers for discontinuation of antithyroid drug treatment in patients with Graves' disease. Clinical Endocrinology 199236 585-590. (doi:10.1111/j.1365-2265.1992.tb02269.x)

70 Allannic H, Fauchet R \& Lorcy Y. A prospective study of the relationship between relapse of hyperthyroid Graves' disease after antithyroid drugs and HLA haplotype. Journal of Clinical Endocrinology and Metabolism 198357 719-722. (doi:10.1210/jcem-57-4-719)

71 Winsa B, Adami HO, Bergstrom R, Gamstedt A, Dahlberg PA, Adamson U, Jansson R \& Karlsson A. Stressful life events and Graves' disease. Lancet 1991338 1475-1479. (doi:10.1016/01406736(91)92298-G)

72 Struja TM, Guebelin L, Kutz A, Fehlberg H, Mueller B \& Schuetz P. Does immunosuppressive therapy improve outcomes in Graves' disease? A systematic review and meta-analysis. Thyroid 201626 634-640. (doi:10.1089/thy.2015.0647)

73 Nygaard B. Hyperthyroidism (primary). BMJ Clinical Evidence 20102010 0605.

74 Vos XG, Endert E, Zwinderman AH, Tijssen JGP \& Wiersinga WM. Predicting the risk of recurrence before the start of antithyroid drug therapy in patients with Graves' hyperthyroidism. Journal of Clinical Endocrinology and Metabolism 2016101 1381-1389. (doi:10.1210/ jc.2015-3644)

Received 24 August 2016

Revised version received 30 September 2016

Accepted 20 October 2016 